\title{
Sarcoidosis, complete heart block, and warm autoimmune hemolytic anemia in a
}

\section{young woman}

\author{
Kate Chernow, BA, ${ }^{\mathrm{a}}$ Timothy Donegan MD, ${ }^{\mathrm{b}}$ Tatyana Milman MD, ${ }^{\mathrm{c}}$ and David Henry \\ $\mathrm{MD}^{\text {de }}$
}

${ }^{a}$ Philadelphia College of Osteopathic Medicine, Pennsylvania; Departments of ${ }^{\mathrm{b}}$ Radiology and ${ }^{\mathrm{d}}$ Medicine, ${ }^{\mathrm{e}}$ Division of Hematology/ Oncology, Pennsylvania Hospital, Philadelphia; and 'Department of Pathology, Hospital of the University of Pennsylvania, Philadelphia

\begin{abstract}
arcoidosis is a multisystem granulomatous disease that affects $10-40$ people per 100,000 in the United States and Europe, with an increased prevalence among blacks compared with whites. ${ }^{1}$ The clinical presentation of sarcoidosis is variable. Sarcoidosis frequently involves the lungs and can have numerous extrapulmonary manifestations including skin, joint, cardiac, and eye lesions. We present a rare case of sarcoidosis with concurrent third-degree heart block and warm autoimmune hemolytic anemia and discuss possible mechanisms behind this presentation.
\end{abstract}

\section{Case presentation}

In September 2014, a 30-year-old white woman with a 3-week history of increasingly frequent dizziness and near syncopal episodes presented to the hospital after 6 episodes of syncope in one day. At the time of admission, she reported having fevers, night sweats, and dark-colored urine for 4 weeks. She had an intentional 100-pound weight loss over the course of 1 year. The patient had a past medical history of polysubstance abuse. During a previous admission for pneumonia in March 2014, a computerized tomography scan of her chest revealed diffuse mediastinal lymphadenopathy and splenomegaly.

On admission, our patient was in third-degree heart block with a junctional escape rhythm and laboratory findings consistent with warm autoimmune hemolytic anemia. The results of a laboratory workup revealed hemoglobin of $7.9 \mathrm{~g} / \mathrm{dL}$ (normal range, female, $12.1-15.1 \mathrm{~g} / \mathrm{dL})$; reticulocyte count, $14.4 \%$ (normal range, 0.5\%-1.5\%); lactate dehydrogenase, $302 \mathrm{U} / \mathrm{L}$ (105-333 U/L); haptoglobin, <30 mg/dL (normal range, female, 12.3-15.3 g/dL); white blood cell count, 5,700/mcL (4,500-10,000/mcL); plate- let count, 261,000/mcL (150,000-400,000/mcL); positive direct and indirect Coombs tests; and positive warm antibodies. Antibody specificity testing showed pan-agglutinin specificity. Spherocytosis was seen on the peripheral smear. Electrolytes were within normal limits. Her liver function tests were elevated: alanine transaminase (ALT), 43 U/L (10$40 \mathrm{U} / \mathrm{L})$; aspartate aminotransferase (AST), $49 \mathrm{U} / \mathrm{L}$ (10-34 IU/L); alkaline phosphatase, $191 \mathrm{U} / \mathrm{L}$ (44$147 \mathrm{IU} / \mathrm{L})$; total bilirubin, $2.7 \mathrm{mg} / \mathrm{dL}(0.3-1.0 \mathrm{mg} /$ $\mathrm{dL})$; direct bilirubin, $0.8 \mathrm{mg} / \mathrm{dL}(0-0.2 \mathrm{mg} / \mathrm{dL})$; and indirect bilirubin, $1.9 \mathrm{mg} / \mathrm{dL}(<1.0 \mathrm{mg} / \mathrm{dL})$.

Her diagnostic work-up was evaluated for infection, malignancy including lymphoma, sarcoidosis, and autoimmune causes. The patient's test results for hepatitis $\mathrm{C}$ virus, human immunodeficiency virus, Lyme disease, rapid plasma reagin for syphilis, tuberculosis, and blood cultures were all negative. She had a normal antinuclear antibody panel. Her serum angiotensin-converting enzyme level was 58 U/L (normal, 8-53 U/L).

The transthoracic echocardiogram was negative for valvular vegetations and showed no structural cause for the third-degree heart block. The patient's chest $\mathrm{x}$-ray showed right hilar and mediastinal lymphadenopathy. The results of a computerized tomography scan of the chest, abdomen, and pelvis showed splenomegaly and mediastinal, hilar, right axillary, and upper abdominal lymphadenopathy along with several new pulmonary nodules (Figure 1). A mediastinal lymph node biopsy revealed noncaseating granulomas consistent with sarcoidosis (Figure 2). The cultures for anaerobic, fungal, and acid-fast bacilli organisms had no growth. Given the patient's diagnosis of sarcoidosis and electrocardiogram findings of third-degree heart block, mag-

Accepted for publication January 17, 2015. Disclosures: The authors have no disclosures. Correspondence: Kate Chernow; katech@pcom.edu. JCSO 2015;13:159-161. @2015 Frontline Medical Communications. DOI 10.12788/jcso.0128. 


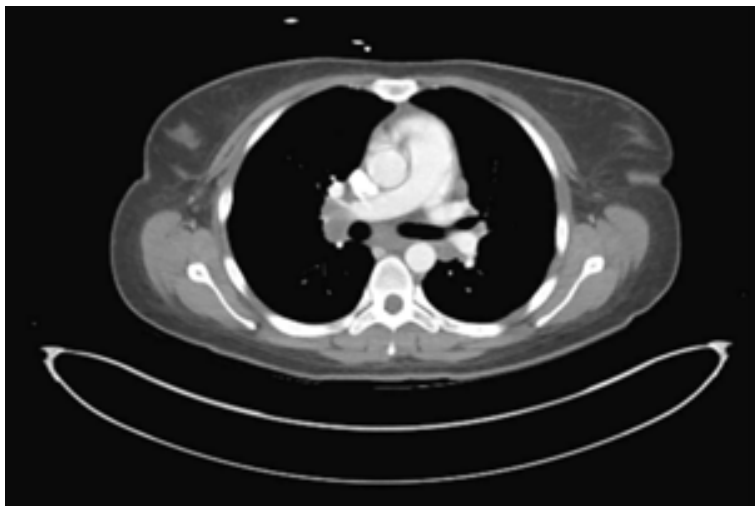

FIGURE 1 Axial computed tomography of the chest, abdomen, and pelvis demonstrates bilateral hilar lymphadenopathy.

netic resonance imaging of the heart was performed and was normal. An ophthalmologic exam showed no signs of ocular involvement from sarcoidosis.

To address her third-degree heart block, a temporary pacemaker was used and subsequently a dual chamber implantable cardioverter defibrillator was placed. The patient was ultimately discharged from the hospital and was prescribed prednisone $60 \mathrm{mg}$ daily for management of her newly diagnosed sarcoidosis. Results from an outpatient cardiac positron-emission tomography scan showed diffuse myocardial glucose uptake, which suggested normal myocardial glucose metabolism. Perhaps, the negative PET scan was the result of the steroid therapy. The warm autoimmune hemolytic anemia resolved with steroids. However, several liver function tests, specifically AST and ALT, were further elevated to $313 \mathrm{U} / \mathrm{L}$ and $528 \mathrm{U} / \mathrm{L}$, respectively.

\section{Discussion}

We present a rare case of sarcoidosis with concurrent thirddegree heart block and warm autoimmune hemolytic anemia. Sarcoidosis is a noncaseating, granulomatous disease of unknown etiology that can involve any organ within the body. We conducted a literature search for similar cases and found 11 reported instances of sarcoidosis with autoimmune hemolytic anemia. Other case reports describe additional associations with thrombocytopenia, ${ }^{2}$ Hodgkin, ${ }^{3}$ non-Hodgkin lymphoma, ${ }^{4}$ and Sjögren's syndrome. ${ }^{5}$ To our knowledge, this is the first reported case of pulmonary sarcoidosis with concurrent third-degree heart block and warm autoimmune hemolytic anemia.

Sarcoidosis is thought to involve a type $1 \mathrm{~T}$-lymphocyte response that is dependent on host susceptibility and characterized by chronic inflammation, monocyte recruitment, and granuloma formation. ${ }^{6}$ Remarkable progress has been made in our understanding of the immunopathogenesis of sarcoidosis. There is now consensus that sarcoidosis is a disease of enhanced, immunologic hypersensitivity to pathogenic tissue antigens. ${ }^{7}$

The clinical outcome of sarcoidosis can vary considerably. Two-thirds of patients with sarcoidosis generally have a remission within a decade after diagnosis, with few or no consequences. However, up to a third of patients have unrelenting disease leading to clinically significant organ impairment. ${ }^{8}$ Treatment of the granulomatous inflammation in sarcoidosis is dependent on nonspecific suppression of the immune response. ${ }^{9}$ Corticosteroids remain the initial treatment of choice for most patients with sarcoidosis due in large part to the clinical variability and high rates of spontaneous remission. ${ }^{9}$ However, several other treatment modalities are commonly used. Although there is limited research in these areas, methotrexate is the most commonly used cytotoxic agent for chronic disease, ${ }^{10}$ and the tumor necrosis factor antibody infliximab has proved useful in treating refractory sarcoidosis. ${ }^{10}$

Of note is that although just over $10 \%$ of patients with sarcoidosis have elevated serum aminotransferase and alkaline phosphatase levels, liver involvement is usually clinically silent. ${ }^{11}$ Our patient had elevation of serum aminotransferase and alkaline phosphatase. In about $5 \%$ of patients with sarcoidosis, granulomas can form in the liver. ${ }^{12}$

Our patient is a young woman with biopsy-confirmed sarcoidosis who also shows clinical manifestations of atrioventricular block. With all other causes being excluded, it is presumed that she has cardiac sarcoidosis. Atrioventricular block is a common finding in patients with clinically evident cardiac sarcoidosis. Prolongation of the PR interval due to disease of the atrioventricular node or bundle of His are common. ${ }^{13}$ Although these lesions may initially be silent, they can progress to complete heart block and cause syncope. ${ }^{14}$ Various definitions and guidelines for diagnosing cardiac sarcoidosis have been proposed. Our patient had a histologic diagnosis of extracardiac sarcoid plus the presence of electrocardiograph abnormalities, which suggests that her heart block is of sarcoid etiology. The most common location for granulomas and scars is the left ventricular free wall, followed by the intraventricular septum, often with involvement of the conducting system. ${ }^{8}$

The treatment of third-degree heart block focuses on treating the underlying cause and frequently involves pacemaker placement. Similarly, implantable cardioverter defibrillator (ICD) therapies in patients with cardiac sarcoidosis are common. An ICD is indicated for some patients with cardiac sarcoidosis for prevention of sudden death. ${ }^{15}$

In addition to cardiac involvement, this patient also developed warm autoimmune hemolytic anemia. Autoimmune hemolytic anemia is a fairly uncommon disorder, with an estimated incidence of 1-3 cases per 100,000 people per year, ${ }^{16}$ which makes the association of warm autoimmune 


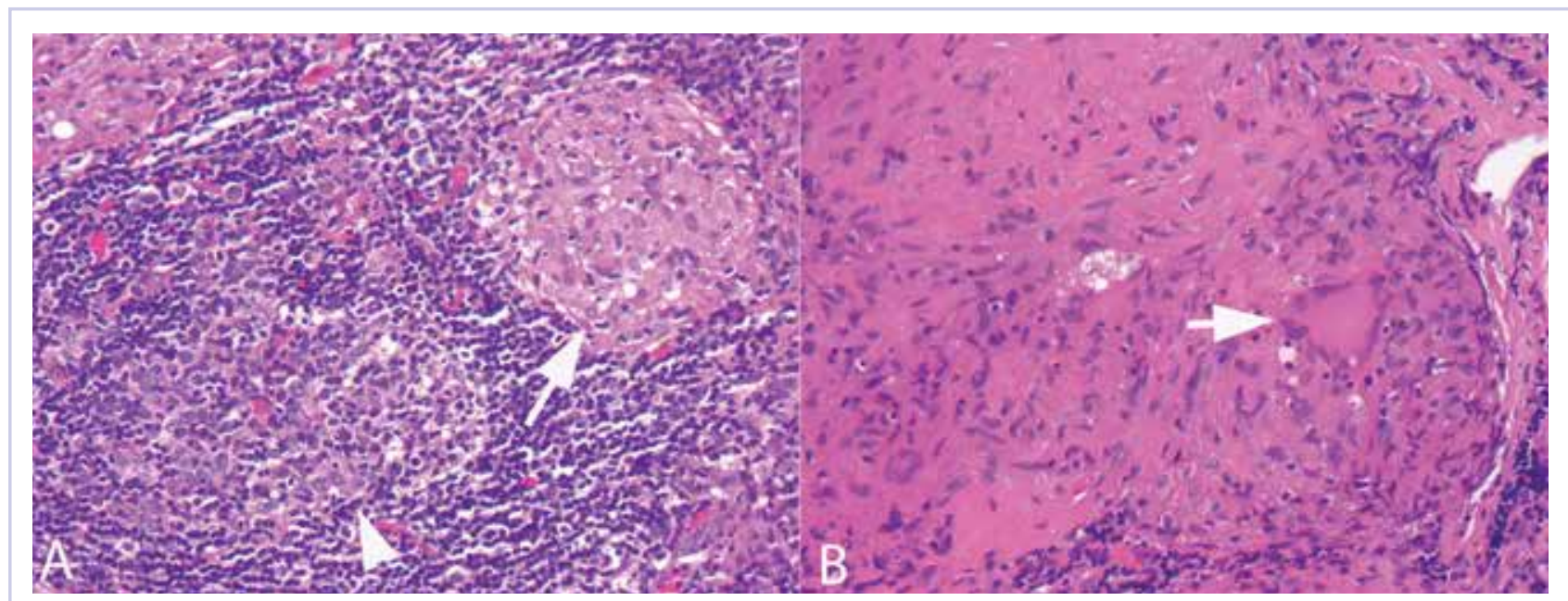

FIGURE 2 A, Lymph node biopsy demonstrates a nonnecrotizing granuloma (arrow) adjacent to a reactive lymphoid follicle (arrowhead; hematoxylin-eosin, original magnification $\times 50$ ). B, High magnification photomicrograph of nonnecrotizing granuloma demonstrates a collection of epithelioid histiocytes and a Langhans-type giant cell (arrow; hematoxylin-eosin, original magnification x100).

hemolytic anemia with sarcoidosis a very rare phenomenon. Warm autoimmune hemolytic anemia occurs because of the presence of immunoglobulin $\mathrm{G}$ antibodies reacting with protein antigens on the surface of red blood cells at normal body temperature. Autoimmune hemolytic anemia is also thought to be the result of altered cell-mediated immunity with inadequate $\mathrm{T}$-suppressor systems causing aberrant humoral immunity. ${ }^{3}$

Treatment of autoimmune hemolytic anemia focuses on reduction of antibody production and antibody effectiveness. The mainstay of treatment of newly diagnosed primary warm autoimmune hemolytic anemia is glucocorticoids. ${ }^{17}$ Steroids help decrease the production of autoantibodies. Second-line therapy includes immunosuppressives, cytotoxic agents, splenectomy, and intravenous gamma globulin. In our patient, oral steroids addressed both her sarcoidosis and her hemolytic anemia.

This young woman's complete heart block seems to be the result of her biopsy-proven sarcoidosis. It is also possible that an immunologic mediator involved in the formation of granulomatous tissue triggered the warm autoimmune hemolytic anemia. In summary, the association of hematologic and other immune disorders with sarcoidosis may reflect general antibody stimulation in sarcoidosis. ${ }^{5}$

\section{References}

1. Rybicki BA, Major M, Popovich J Jr, Maliarik MJ, Iannuzzi MC Racial differences in sarcoidosis incidence: a 5-year study in a health maintenance organization. Am J Epidemiol. 1997;145:234-241.

2. Schneider RM, Worsley A, Lichtman S, Meyer RJ. Sarcoidosis with immune hemolytic anemia and thrombocytopenia: Humoral aberrations responding to steroids or splenectomy. Mt Sinai J Med. 1982;49:115-120.
3. Spitzer T, Crum E, Schacter L, Abboud S. Sarcoidosis, hodgkin's disease, and autoimmune hemolytic anemia. Am J Med Sci. 1986;291:190-193.

4. Taillan B, Pedinielli FJ, Routy JP, Blanc AP. Non-hodgkin malignant lymphoma and autoimmune anemia during the development of sarcoidosis [in French]. Rev Med Interne. 1985;6:573-575.

5. Kondo H, Sakai S, Sakai Y. Autoimmune haemolytic anaemia, Sjögren's syndrome and idiopathic thrombocytopenic purpura in a patient with sarcoidosis. Acta Haematol. 1993;89:209-212.

6. Hunninghake GW, Gadek JE, Young RC Jr, Kawanami O, Ferrans VJ, Crystal RG. Maintenance of granuloma formation in pulmonary sarcoidosis by $\mathrm{T}$ lymphocytes within the lung. $\mathrm{N}$ Engl $\mathrm{J}$ Med. 1980;302:594-598.

7. Mitchell D, Wells A, Spiro S, Moller D, eds. Sarcoidosis. London, UK: Hodder Arnold; 2012.

8. Iannuzzi MC, Rybicki BA, Teirstein AS. Sarcoidosis. N Engl J Med. 2007;357:2153-2165.

9. Lazar CA, Culver DA. Treatment of sarcoidosis. Semin Respir Crit Care Med. 2010;31:501-518.

10. Baughman RP, Costabel U, du Bois RM. Treatment of sarcoidosis. Clin Chest Med. 2008;29:533-548.

11. Baughman RP, Tierstein AS, Judson MA, et al; Case Control Etiologic Study of Sarcoidosis (ACCESS) research group. Clinical characteristics of patients in a case control study of sarcoidosis. Am J Respir Crit Care Med. 2001;164:1885-1889.

12. Scott GC, Berman JM, Higgins JL Jr. CT patterns of nodular hepatic and splenic sarcoidosis: a review of the literature. J Comput Assist Tomogr. 1997;21:369-372.

13. Chapelon-Abric C, de Zuttere D, Duhaut P, et al. Cardiac sarcoidosis: A retrospective study of 41 cases. 2004;83:315-334.

14. Yoshida Y, Morimoto S, Hiramitsu S, Tsuboi N, Hirayama H, Itoh T. Incidence of cardiac sarcoidosis in Japanese patients with highdegree atrioventricular block. Am Heart J. 1997;134:382-386.

15. Schuller JL, Zipse M, Crawford T, et al. Implantable cardioverter defibrillator therapy in patients with cardiac sarcoidosis. J Cardiovasc Electrophysiol. 2012;23:925-929.

16. Gehrs BC, Friedberg RC. Autoimmune hemolytic anemia. Am J Hematol. 2002;69:258-271.

17. Lechner K Jäger U. How I treat autoimmune hemolytic anemias in adults. Blood . 2010;116:1831-1838. 